\title{
Single ionization of atoms in intense laser pulses: Evolution from multiphoton to tunnel ionization
}

\author{
A. Rudenko ${ }^{* a}$, K. Zrost ${ }^{\mathrm{a}}$, Th. Ergler ${ }^{\mathrm{a}}$, B. Feuerstein ${ }^{\mathrm{a}}$, V.L.B. de Jesus ${ }^{\mathrm{b}}$, C.D. Schröter ${ }^{\mathrm{a}}$, \\ R. Moshammer ${ }^{\mathrm{a}}$ and J. Ullrich ${ }^{\mathrm{a}}$ \\ ${ }^{a}$ Max-Planck-Institut für Kernphysik, D-69029 Heidelberg, Germany \\ ${ }^{\mathrm{b}}$ Centro Federal de Educação Tecnológica de Química de Nilópolis/RJ, \\ Rua Lucio Tavares 1045, Centro - Nilópolis - 26530-060, Rio de Janeiro, Brazil
}

\begin{abstract}
We present results of high resolution fully differential measurements on single ionization of $\mathrm{He}$, Ne, and Ar by 7 - 25 fs linearly polarized $800 \mathrm{~nm}$ laser pulses at intensities of up to $2 \cdot 10^{15} \mathrm{~W} / \mathrm{cm}^{2}$. Using a 'Reaction-Microscope' we were able to trace signatures of multiphoton ionization deep into the tunnelling regime. Surprisingly, in the low-energy electron spectra we observed several features (absence of the ponderomotive shifts, splitting of the peaks, their degeneration for few-cycle laser pulses) typical for resonantly-enhanced ionization. Other remarkable features, as the sharp cusp-like momentum distributions in the direction perpendicular to the laser field or the observed minima at zero longitudinal momentum for $\mathrm{He}$ and $\mathrm{Ne}$, can be reproduced by semiclassical models, where the electron motion in the combined laser and Coulomb field is treated classically after the tunnelling.
\end{abstract}

Keywords: multiphoton processes, tunneling, above-threshold ionization, ponderomotive shift, Coulomb cusp.

\section{INTRODUCTION}

Single electron emission from atoms exposed to intense short-pulse laser fields is one of the most fundamental and wellstudied reaction in non-linear strong-field physics ${ }^{1-3}$. One of the basic questions, however, which still disturbs a comprehensive understanding of the ionization dynamics, concerns the intensity regime that might be characterised by the transition from a "photon" to a "field" based view of the problem. Usually, ionization is considered as either a "multiphoton" or "tunnelling" process depending on the value of the so-called Keldysh parameter ${ }^{4}=\sqrt{I_{p} / 2 U_{p}}$ (where $I_{p}$ is the ionization potential and $U_{p}=I / 4 \omega^{2}$ the ponderomotive potential. $I$ is the light intensity and $\omega$ its frequency; atomic units are used throughout if not stated otherwise). For $\gamma>1$ numerous experiments revealed the multiphoton nature of ionization reflected in a rich structure in the photoelectron spectra. Various field induced processes, such as resonant and non-resonant above-threshold ionization (ATI), ponderomotive effects, channel opening and closing etc. were studied, and good agreement between theory and experiment has been achieved ${ }^{5-10}$.

For the other extreme case of very low $\gamma$ at high intensities only few experimental results have been reported. Early experiments performed with low-frequency radiation ${ }^{11}$ showed a good agreement with a quasistatic tunnelling model ${ }^{12-}$ 13. Within this model it is assumed that the electron first tunnels into the continuum and then moves classically driven by the oscillating laser field. For the case of linearly polarized light the motion of atomic or molecular fragments emerging from ionization can be naturally separated into a longitudinal part, which is parallel to the electric field

*a.rudenko@mpi-hd.mpg.de phone: +496221516609 fax: +496221516604 
polarization, and a part transverse to the field. Under these assumptions analytical expressions for the momentum distributions along $\left(\mathrm{W}\left(\mathrm{P}_{\|}\right)\right)$and perpendicular $(\mathrm{W}(\mathrm{Px}, \mathrm{y}))$ to the field polarization were obtained ${ }^{11-13}$ :

$$
\begin{aligned}
& W\left(P_{\|}\right) \sim \exp \left[-(1 / 3) P_{\|}^{2} \omega^{2}\left(2 I_{p} / I\right)^{3 / 2}\right], \\
& W\left(P_{x, y}\right) \sim \exp \left[-P_{x, y}^{2}\left(2 I_{p} / I\right)^{1 / 2}\right] .
\end{aligned}
$$

(Here $\mathrm{P}_{\mathrm{x}, \mathrm{y}}$ are the two transverse momentum components.) Later, experiments at optical frequencies observed a significant fraction of electrons with energies higher than the maximal drift energy of $2 U_{p}$ that an electron can gain in the field, forming a plateau-like structure in the high-energy part of the electron spectra ${ }^{14-15}$. Since then, efforts strongly concentrated to explore this feature, which has been explained within the tunnelling picture by considering the return of the electron to the ion core and subsequent elastic rescattering ${ }^{14}$. In contrast, for the low-energy part of the spectrum first experiments, where ion (and thus, indirectly, electron) momentum distributions for single ionization were recorded, showed reasonable agreement with the predictions of Eq. $(1,2)$ both, for longitudinal and transverse directions ${ }^{16,17}$. The situation surprisingly changed when the experimental resolution was improved. Recent high-resolution measurements on single ionization clearly showed that longitudinal distributions at zero momentum deviate from the smooth Gaussian shape $^{18-20}$. For the case of He this was predicted within the semiclassical model ${ }^{21}$, where tunnelling with subsequent classical propagation of the emitted electron in the combined laser and Coulomb fields was considered. In the same work the authors predicted some deviations from the Gaussian shape also for the transverse momentum distribution.

Recently, high-resolution experiments ${ }^{22,23}$ provided evidences for the resonant origin of detailed narrow structures within the plateau, in agreement with a series of numerical simulations ${ }^{24,25}$. A comprehensive numerical analysis of the problem $^{25}$ revealed a close relation between resonant-like behaviour within the plateau and the existence of electron trajectories which lead to multiple recollisions with the parent ion. The significance of multiphoton resonances for "super-ponderomotive" photoelectrons in the tunnelling regime was predicted for the case of $\mathrm{He}^{26}$, but was never confirmed experimentally. The lowest value of the Keldysh parameter for which some regular ATI structure was ever observed is, to the best of our knowledge, $\gamma \sim 0.7^{9,14}$. Experiments performed at lower $\gamma$ either revealed a smooth decrease of the photoelectron spectra, with changes of the slope in the plateau region ${ }^{15}$, or observed in addition some hardly resolved structure below $20 \mathrm{eV}^{9,27}$.

In this paper we report on the experimental observation of well resolved pronounced and regular pattern in the electron emission characteristics for single ionization of $\mathrm{He}, \mathrm{Ne}$ and $\mathrm{Ar}$ at intensities of up to $1.5 \cdot 10^{15} \mathrm{~W} / \mathrm{cm}^{2}(\gamma<0.5)$. We show that for 25 fs laser pulses the low-energy part of the photoelectron spectra consists of a series of wide peaks separated by the photon energy, which exhibit features (absence of ponderomotive shifts, narrow substructures resolved within different peaks) typical for resonantly-enhanced ionization. These features are considerably washed out for 6-7 fs pulses. For ultra-low energy electrons clear signatures of the atomic structure can be observed in the spectra, while for higher energies the results look similar for different atomic species. We also present high-resolution transverse momentum distributions. In contrast to Eq. (2) the spectra exhibit a sharp cusp-like structure around zero transverse momentum. Comparing our experimental data with theoretical results, we conclude that the observed singularity originates from the Coulomb interaction between the emitted electron and its parent ion. We found a striking similarity to the cusps observed in ion-atom collisions.

The paper is structured as follows: Section 2 gives a brief description of the experimental setup. The experimental results are presented and discussed in Section 3 and conclusions are given in Section 4.

\section{EXPERIMENTAL SETUP}

Fig. 1 shows a sketch of the experimental arrangement. Measurements were performed using a newly designed "reaction microscope" 28 with further improved momentum resolution along the time-of-flight direction $(\Delta \mathrm{P}<0.02$ a.u. $)$. We used linearly polarized radiation of a Kerr-lens mode locked Ti:sapphire laser at $795 \mathrm{~nm}$ wavelength amplified to pulse energies of up to $350 \mu \mathrm{J}$ at $3 \mathrm{kHz}$. The width of the amplified pulses was $25 \mathrm{fs}$. To generate few-cycle pulses 


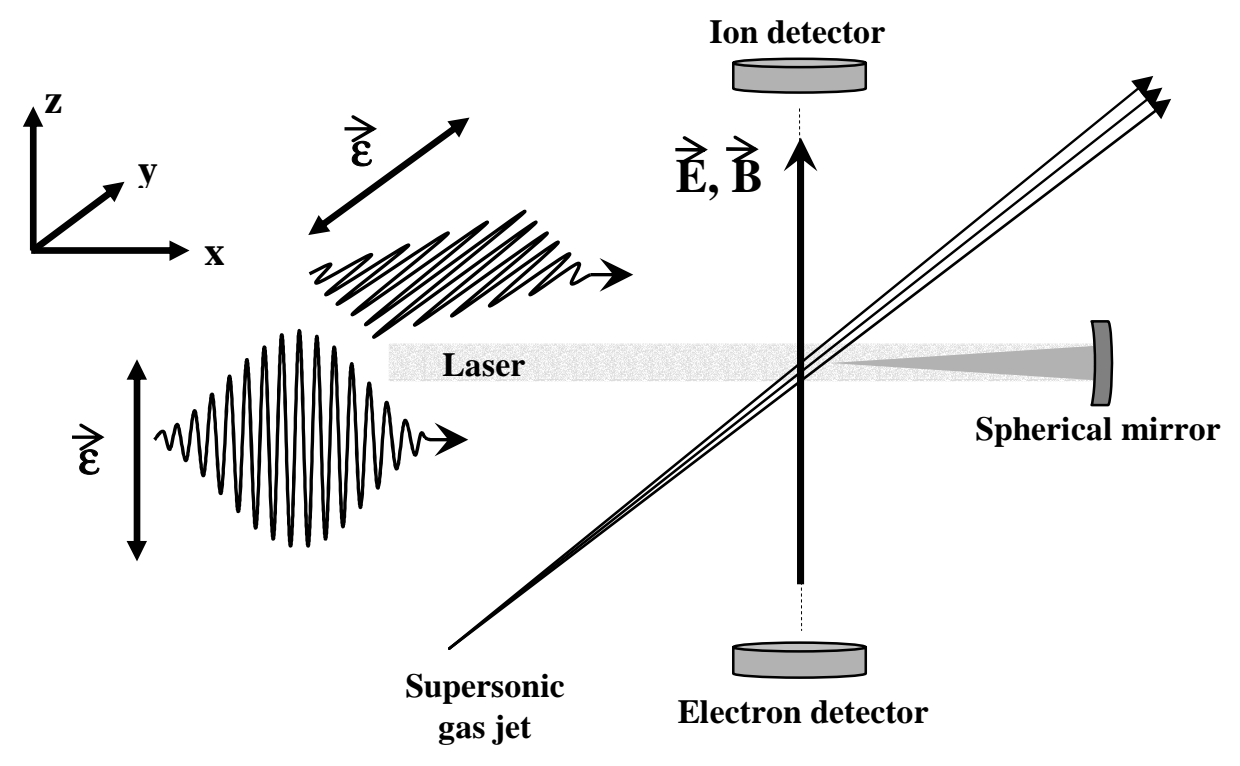

Figure 1. Sketch of the experimental setup. The laser polarization was oriented either parallel to the timeof-flight axes, or perpendicular to it for high-resolution measurements of the longitudinal or the transverse momentum, respectively.

they were spectrally broadened in a gas-filled hollow fiber and then compressed to 6-7 fs by chirped mirrors and a prism compressor. The laser beam was focused to a spot size of $\sim 7 \mu \mathrm{m}$ onto the collimated supersonic gas jet in the ultra-high vacuum chamber $\left(2 \cdot 10^{-11} \mathrm{mbar}\right)$. Fluctuations of the laser intensity from pulse to pulse were monitored during the experiment and did not exceed 5\%. Absolute calibration of the peak intensity was performed using a clear kink in the measured momentum distribution of $\mathrm{Ne}^{+}$ions, which corresponds to the maximum drift momentum of $2 \sqrt{U_{p}}$ that electrons can gain from the laser field ${ }^{29}$. Comparing the intensity obtained from this calibration with those obtained by measuring the $10 U_{p}$ cutoff $^{30}$, by analyzing the momentum distribution for circularly polarized light ${ }^{31}$, and by calculating the intensity using the measured optical parameters of the laser beam, we estimate the error of our calibration to be less than $15 \%$.

Ions and electrons were guided to two position-sensitive channel plate detectors by weak electric $(1 \mathrm{~V} / \mathrm{cm})$ and magnetic (5G) fields. From the time of flight and position the full momentum vectors of coincident ions and electrons were calculated. Neglecting the small momentum of the absorbed photons, the momentum distributions of electrons and ions for single ionization should be just mirror images of each other, provided the experimental resolution is the same. Momentum conservation along the time-of-flight axis was ensured for each event. This allows one to exclude possible influences of space-charge effects or ponderomotive acceleration in the spatially inhomogeneous laser field since both would act differently on electrons and ions. It also ensures that absolutely no contributions from higher charge states or impurities from the residual gas enter the spectra. In order to investigate the longitudinal and transverse momenta of the fragments with the best achievable resolution we performed the experiment in two different modes, employing geometries where the laser polarization was oriented either parallel to the extraction field (z direction) and, correspondingly, perpendicular to the direction of the target gas jet (y direction), or, vice versa, parallel to the jet and perpendicular to the extraction field. Thus, either the longitudinal momentum or one component of the transverse momentum could be obtained from the measured time-of-flight, allowing for a momentum resolution in the direction of interest of better than 0.02 a.u. In the following we denote the longitudinal momentum as $\mathrm{P}_{\|}$, the two components of the transverse momentum as $\mathrm{P}_{\mathrm{x}}$ and $\mathrm{P}_{\mathrm{y}}$, and the full transverse momentum as $\mathrm{P}_{\perp}=\left(\mathrm{P}_{\mathrm{x}}{ }^{2}+\mathrm{P}_{\mathrm{y}}{ }^{2}\right)^{1 / 2}$. It should be noted that since for the linear polarized light the system is cylindrically symmetric with respect to the polarization axes, the distributions $\mathrm{W}\left(\mathrm{P}_{\mathrm{x}}\right)$ and $\mathrm{W}\left(\mathrm{P}_{\mathrm{y}}\right)$ should be identical. This was confirmed by inspection of the measured electron momentum distributions. 


\section{RESULTS AND DISCUSSION}

\subsection{Longitudinal momentum distributions}

Fig. 2 displays longitudinal momentum distributions of singly charged ions created by 25 fs laser pulses for the case of He (Fig. 2a) and Ar (Fig. 2b) at different intensities ( $\gamma$ varies from 1.1 to 0.29), whereas Fig. 3 shows longitudinal momentum distributions for Ne obtained with 25 and 7 fs pulses.. The data are integrated over the transverse momenta.

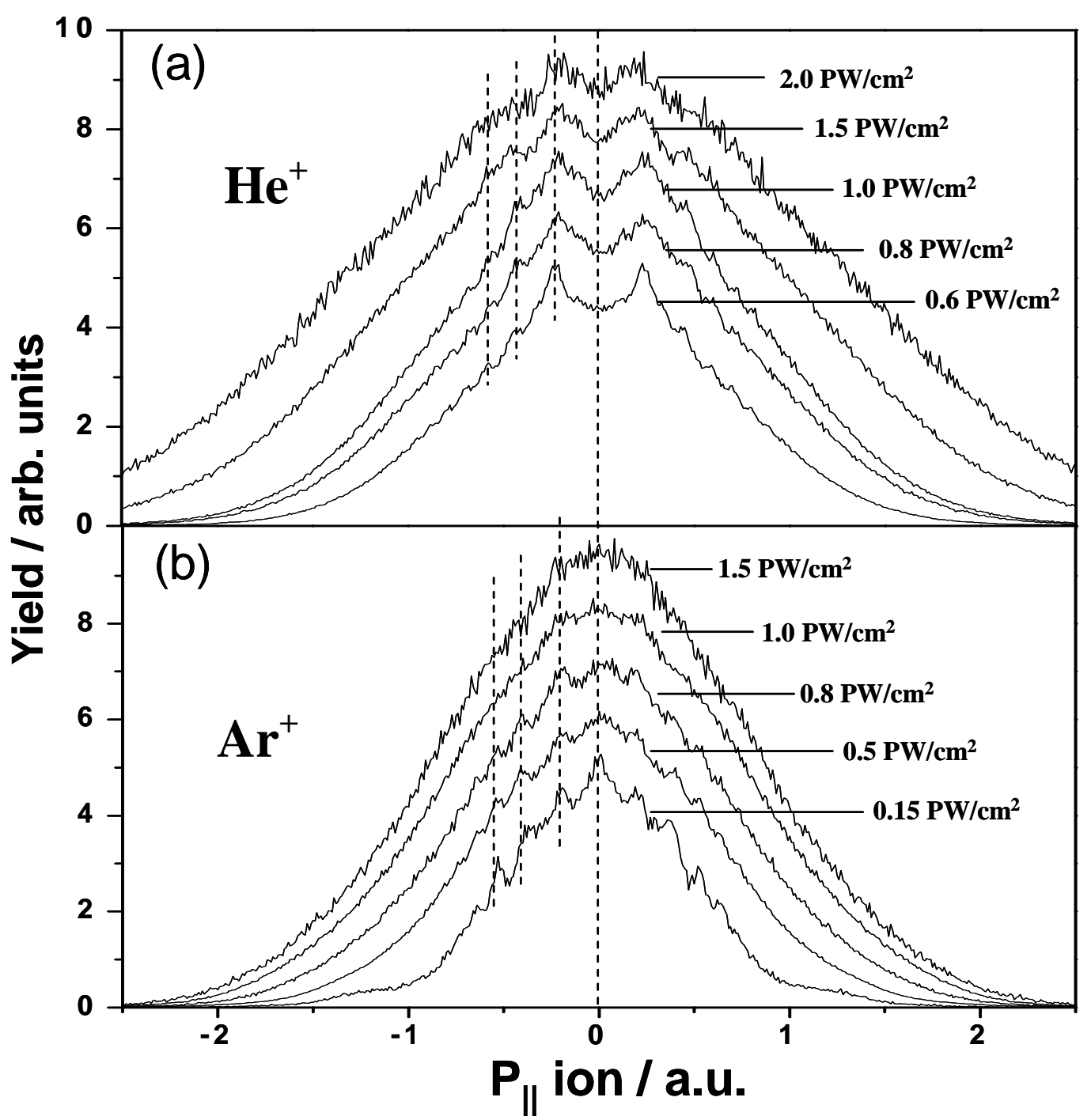

Figure 2. Momentum distributions of singly charged $\mathrm{He}$ (a) and $\mathrm{Ar}$ (b) ions along the laser polarization direction for 25 fs laser pulses.

For all three targets the spectra for 25 fs pulses at the lowest intensity manifest a clear set of peaks that are broadened as the intensity grows, emerging for the highest intensities into a shoulder-like structure at the positions of the first two peaks. Several surprising features can be observed. First, at least remnants of the lowest order peaks can be clearly followed up to the highest intensities, deep in the tunnelling regime. Second, all peak positions are independent of the intensity. Third, clear remnants of the peaks can be observed even for the $7 \mathrm{fs}$ pulses, although they are considerably smoothed. 


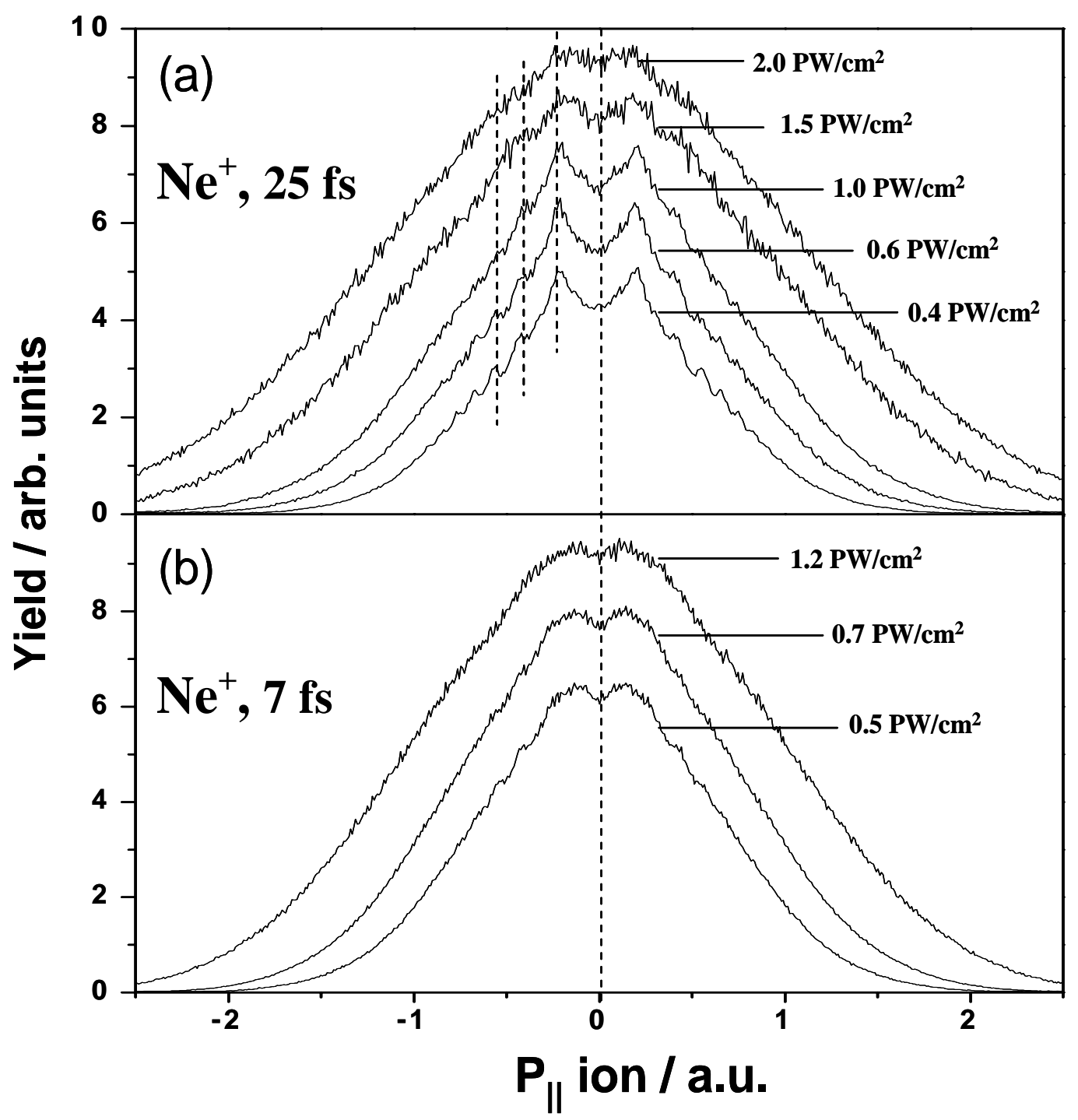

Figure 3. Momentum distributions of singly charged Ne ions along the laser polarization direction for 25 (a) and 7 (b) fs laser pulses.

Apart from the overall similarity of the observed pattern for all the targets there is one striking difference, the appearance of a pronounced minimum at zero longitudinal momentum for $\mathrm{He}$ and $\mathrm{Ne}$, whereas there is a clear maximum for Ar. Within semiclassical calculations ${ }^{21,32}$, the dip at zero momentum was attributed to the interaction of electrons with their parent ion through many recollisions at very low energy. Accordingly, the shape of the scattering potential should influence the emission of ultra-low energy electrons and therefore they might be the only ones that remain sensitive to the target structure at very high intensities.

\subsection{Electron energy spectra and angular distributions}

In order to clarify the origin of the observed structures, we next examine the electron energy spectra. As shown in Fig. 4 for the case of $\mathrm{Ne}$, the electron energy distributions also exhibit a series of peaks, which are separated by the photon energy and broadened with increasing intensity. Thus, we do observe the evolution of the ATI-like structure and again, as in the momentum representation, the peaks do not exhibit intensity-dependent shifts (see vertical dashed lines in Fig. 2-4). Zooming into the details by fully exploiting the high resolution of our electron imaging system along the 


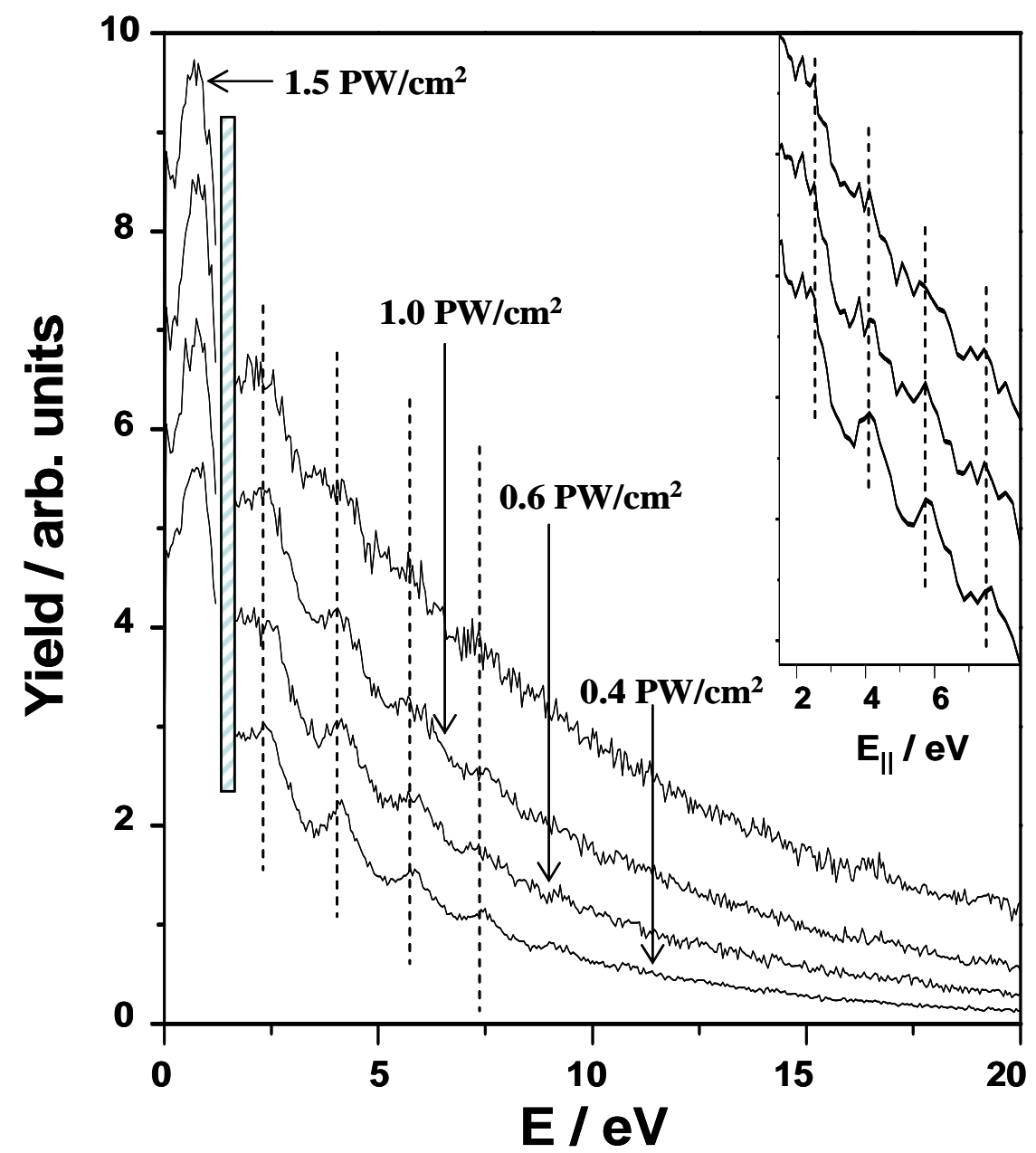

Figure 4. Electron energy distributions for single ionization of Ne by $25 \mathrm{fs}$ pulses at different intensities. The data are integrated over all emission angles. Vertical cut shows the region where the spectrometer has no resolution in the transverse direction. Inset: longitudinal component of the kinetic energy. The intensities correspond to the three lowest curves in the main figure.

longitudinal direction and plotting the longitudinal part of the electron kinetic energy only, we find a pronounced fine structure with at least two sub-peaks repeated within different ATI orders at different intensities (see the inset of Fig. 4).

The splitting of the ATI-like peaks together with the absence of any detectable ponderomotive shifts might lead to assume them to be of resonant nature. Non-resonant ATI peaks are known to shift towards lower energies as the intensity grows due to the field-induced increase of the ionization potential, which is equal to the ponderomotive energy $\mathrm{U}_{\mathrm{p}}{ }^{10,33}$. For $\lambda=795 \mathrm{~nm}$ at a laser peak intensity of $1.0 \mathrm{PW} / \mathrm{cm}^{2} \mathrm{U}_{\mathrm{p}}$ is as high as $60 \mathrm{eV}$. Thus, averaging over different intensities (spatio-temporal pulse distribution and intensity fluctuations) on a level of less than $3 \%$ will result in variations of $U_{p}$ exceeding the photon energy and, thus, any non-resonant ATI peaks will definitely be smeared out.

The situation is different for the case of resonantly-enhanced ionization, which occurs through an excited atomic level shifted into multiphoton resonance with the ground state. In early studies two scenarios resulting in the intensityindependent ATI peak positions were suggested. The first one ${ }^{7}$ assumes that ionization from an excited state occurs before the intensity has considerably changed, so that it always proceeds at the intensity required to fulfil the resonance condition and accordingly, at some certain value of the ponderomotive shift. If the peak intensity is higher than the 


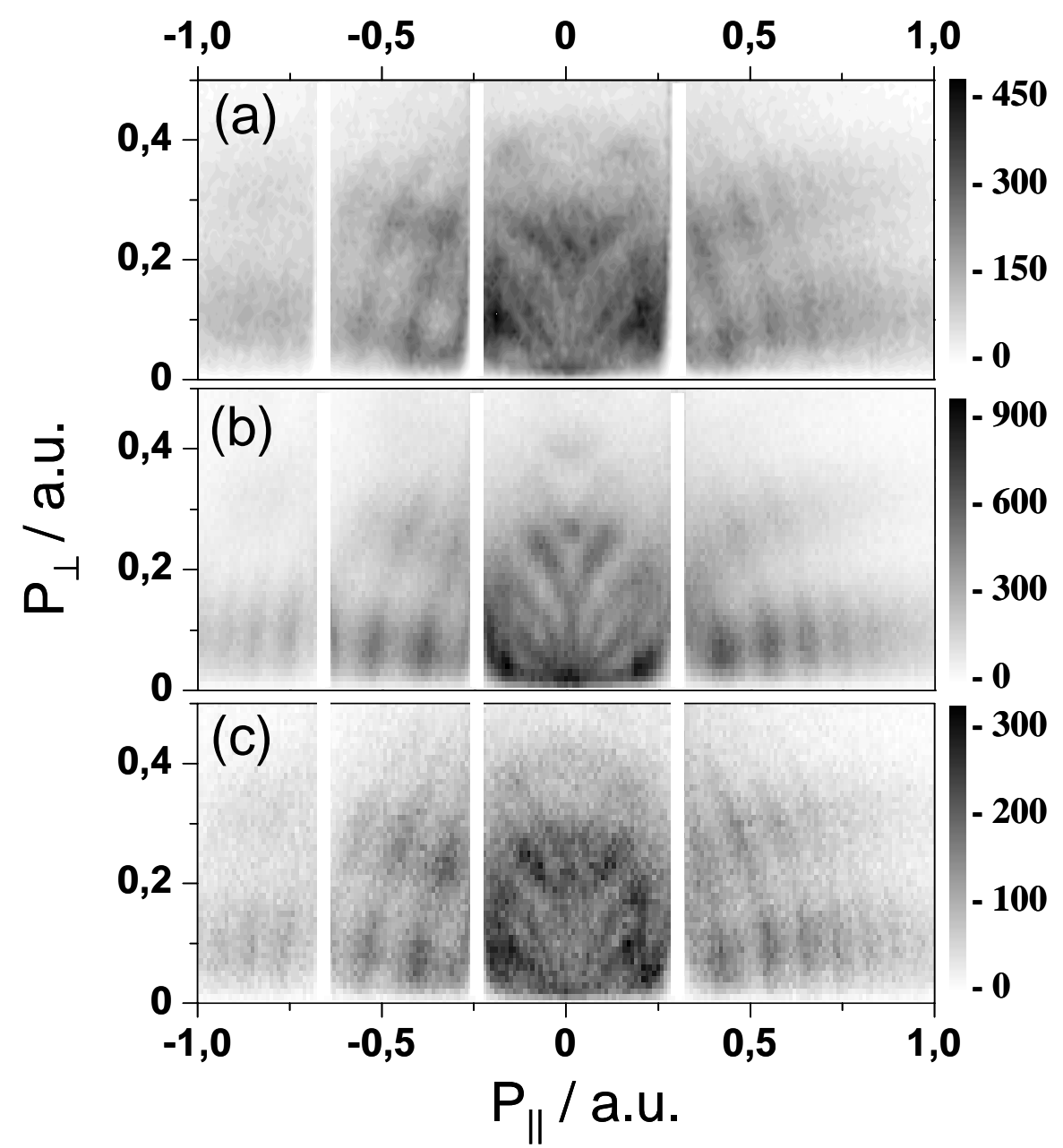

Figure 5: Electron momentum distribution parallel $\left(P_{\|}=P_{z}\right)$ and perpendicular $\left(P_{\perp}=\sqrt{P_{x}^{2}+P_{y}^{2}}\right)$ to the laser polarization direction. (a) He, $25 \mathrm{fs}, 0.6 \mathrm{PW} / \mathrm{cm}^{2}$. (b) $\mathrm{Ar}, 25 \mathrm{fs}, 0.5 \mathrm{PW} / \mathrm{cm}^{2}$. (c) Ne, $25 \mathrm{fs}, 0.6 \mathrm{PW} / \mathrm{cm}^{2}$. Vertical cuts show regions where the spectrometer has no resolution in the transverse direction.

resonant value, the resonance condition can be fulfilled somewhere in the laser focus. The second scenario ${ }^{8}$ suggests that the excited state survives longer in the laser pulse and is ionized later by one- or multi-photon absorption. If the excited state is a high-lying Rydberg state, it is shifted upwards almost as much as the continuum level, again giving rise to intensity-independent peak positions. Of course, such considerations can hardly be directly applied at high. intensities, where any intermediate state must be completely deformed by the laser field. However, numerical simulations ${ }^{24-26}$ have shown that some excited states may remain surprisingly stable or even be induced by the laser field (light induced states). The part of the wave function that tunnels out of the atom might effectively populate these extended quasi-bound states producing resonant-like structures in the photoelectron spectra ${ }^{23,26}$.

Contributions from any resonant-like mechanism should be sensitive to the length of the laser pulse. For pulse durations of less than 10 fs all high-lying Rydberg states have Kepler periods considerably longer, which can result in a suppression of resonantly-enhanced ionization ${ }^{30}$. Besides that, if the lifetime of the excited state is comparable to the pulse length, their mutual interrelation starts to play a role. In our data, comparing results obtained with $25 \mathrm{fs}$ and 6-7 fs pulses for Ne (Fig. 3a and 3b), one can see that with shorter pulses the ATI peaks are smoothed out, and are only distinguishable for the lowest intensity. 
Even more information about the ionization dynamics can be obtained by consideration of the angular-resolved data. Fig. 5 presents density plots of two-dimensional electron momentum distributions for different atomic species and pulse lengths. In this representation ATI peaks manifest themselves as ring-like structures. In striking contrast to the common belief, the electron emission characteristics show a surprisingly rich structure, even in the tunneling regime. The spectra clearly contain target-specific features in the low-energy region; however, for higher longitudinal momenta the spectra for different targets exhibit similar pattern with pronounced maxima and minima in the angular distribution. As of now, the origin of the structures observed in the two-dimensional momentum distributions in terms of simple physical pictures remains completely unclear. The angular distribution of any resonant-enhanced contribution should reflect the symmetry of the state through which ionization has occurred ${ }^{10,33}$. This can result in an atomic species dependence of the data, in particular, in different behaviour in the ultra-low energy region, as observed in Fig. 2-3 and Fig. 5. Taking into account the low peak-to-valley contrast of the spectra, we assume that the resonantly enhanced part is embedded into a strong non-resonant contribution, and thus, superposition of both should define the angular distribution of the emitted electrons.

\subsection{Transverse momentum distributions: signatures of the Coulomb singularity}

In order to perform a detailed comparison between theory and experiment one should not only account for contributions from resonant and non-resonant intensities realized in different parts of the spatio-temporal distribution of the pulse, but also properly describe the atomic structure modified by the laser field. This way single ionization of Ar at intensities up to $10^{14} \mathrm{~W} / \mathrm{cm}^{2}$ was perfectly described within a single active electron model by solving numerically the time-dependent Schrödinger equation ${ }^{10}$. The extension of this approach to higher intensities would, however, require larger computational efforts, and it is definitely beyond present computing capabilities for many-electron reactions. On the other hand, semiclassical models considering tunnelling with subsequent electron motion in the combined laser and Coulomb fields, which provide a satisfactory description of some basic features of single and double ionization ${ }^{21,32,34}$ have obvious limitations due to their classical nature. Thus, a proper description of the ionization process within other theoretical frameworks, in particular, the strong-field approximation (SFA), known also as Keldysh-Faisal-Reiss approach $^{4,35,36}$, was one of the major challenges for theory over the last two decades.

Within the latter approach one of the basic problems is a proper treatment of the Coulomb interaction between the electron and the nucleus ${ }^{37-41}$. Experimentally, to explore the role of this interaction in the ionization process, the analysis of electron and ion transverse momenta is particularly suited, since the laser field (neglecting its magnetic component) does not act in the transverse direction. This was recently exploited ${ }^{42}$ in order to study the final state Coulomb interaction between two electrons in strong-field double ionization. Here we use this analysis to explore Coulomb effects in single ionization.

Fig. 6 shows measured electron transverse momentum distributions for single ionization of $\mathrm{He}$, Ne and $\mathrm{Ar}$ by 25 fs (a-c) and $7 \mathrm{fs}(\mathrm{d})$ laser pulses. Note that only one component of the transverse momentum is shown here, in contrast to Fig. 5. As can be seen from Fig. 6c,d, there is no major differences between the results obtained with 25 and $7 \mathrm{fs}$ pulses. The spectra for different targets look similar for $\left|\mathrm{P}_{\mathrm{x}}\right|<0.5$ a.u., exhibiting a characteristic sharp non-Gaussian shape with a slight change of the slope around \pm 0.25-0.3 a.u.. At higher momenta another change of the slope can be observed, which is most pronounced for Ne.

In Fig. 7 the experimental data for He are compared with theoretical results. The dotted line represents results ${ }^{43}$ of a SFA calculation (in its form introduced by Reiss ${ }^{36}$ ), in which, however, the electron final state was modeled by a Coulomb-Volkov ${ }^{40,41}$ rather than a Volkov state. These results were averaged over the intensity distribution in the laser focal volume and over the time profile of the laser pulse. The theoretical results are in good agreement with the experiment, exhibiting the characteristic cusp-like structure at $\mathrm{P}_{\mathrm{x}}=0$. Note that this structure does not appear if the final state electron wave function is approximated by a Volkov state.

Fig. 7 also displays results of classical trajectory Monte Carlo (CTMC) simulations ${ }^{32}$, where the CTMC approach was modified to allow for tunnelling (CTMC-T). Within this approach the numerical integration of the classical equations of motion allows one to trace explicitly the influence of the Coulomb interaction between an electron and its parent ion on the resulting momentum distribution. As shown in Fig. 7, if this interaction is taken into account, the simulations nicely reproduce the experimental results. However, if the Coulomb interaction is switched off, a Gaussian spectrum shape is restored, in accordance with Eq. (2). 


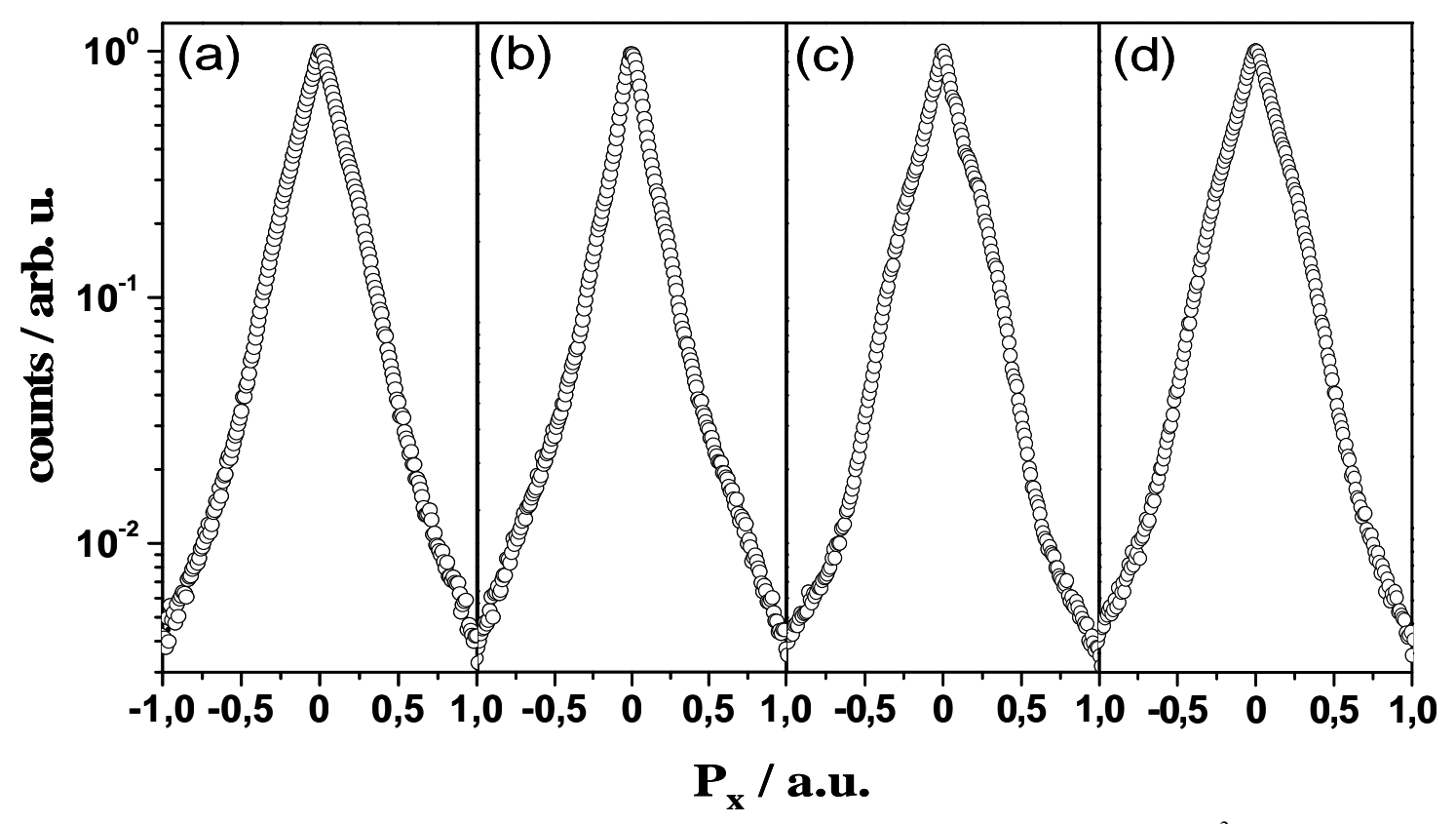

Figure 6. Transverse momentum distributions for single ionization. (a) $\mathrm{He}, 25 \mathrm{fs}, 0.6 \mathrm{PW} / \mathrm{cm}^{2}$. (b) Ar, $25 \mathrm{fs}$, $0.25 \mathrm{PW} / \mathrm{cm}^{2}$. (c) Ne, $25 \mathrm{fs}, 0.6 \mathrm{PW} / \mathrm{cm}^{2}$. (d) $\mathrm{Ne}, 7 \mathrm{fs}, 0.7 \mathrm{PW} / \mathrm{cm}^{2}$.

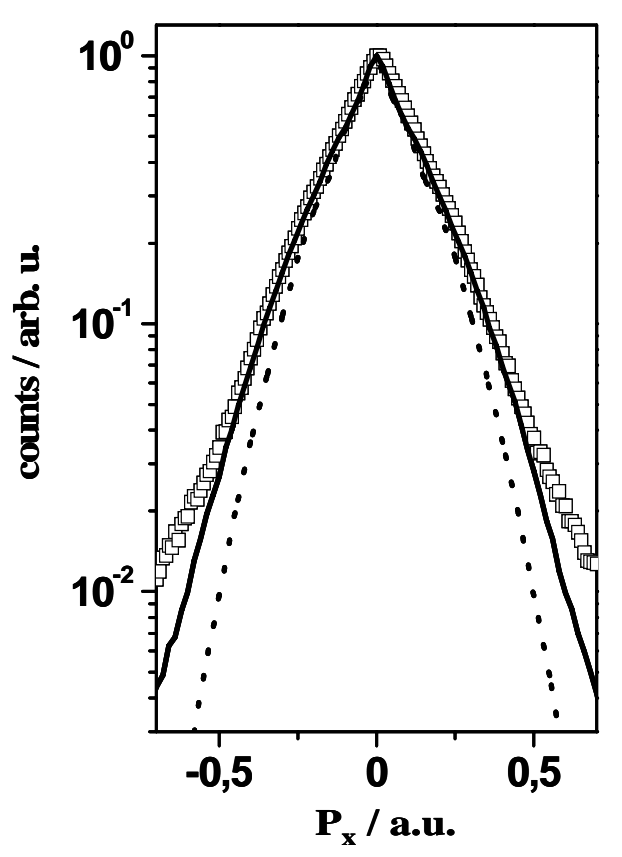

Figure 7. Measured and calculated transverse momentum distributions for single ionization. Sqares: experiment, He, 0.6 $\mathrm{PW} / \mathrm{cm}^{2}$. Dotted line: The SFA calculation ${ }^{43}$ with the

Coulomb-Volkov final state wave function, $\mathrm{H}, 0.6 \mathrm{PW} / \mathrm{cm}^{2}$. Solid line: CTMC-T calculations ${ }^{32}$ with Coulomb potential, $\mathrm{H}, 0.35 \mathrm{PW} / \mathrm{cm}^{2}$.

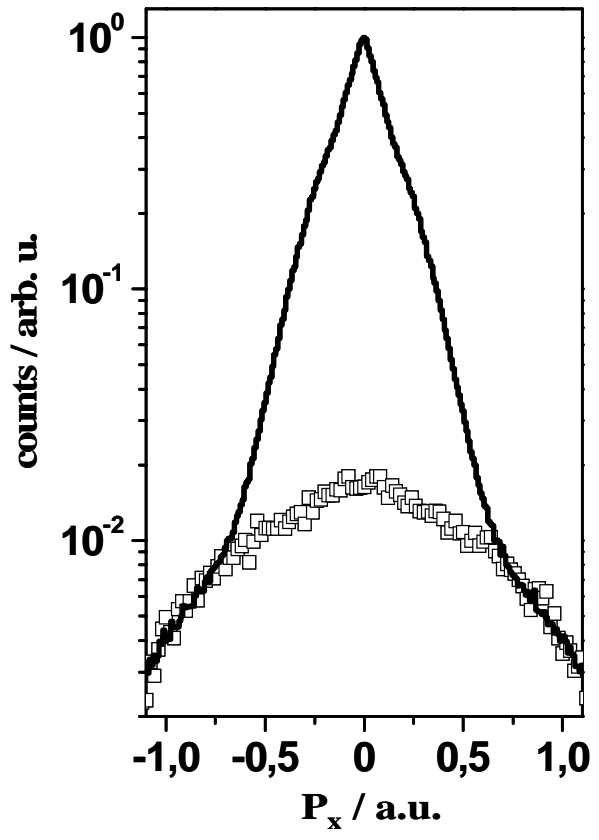

Figure 8. Transverse momentum distributions for single ionization of $\mathrm{Ne}$ by $25 \mathrm{fs} 0.6 \mathrm{PW} / \mathrm{cm}^{2}$ laser pulses. Solid line: all electrons; sqares: electrons with longitudinal momenta larger than $2\left(\mathrm{U}_{\mathrm{p}}\right)^{1 / 2}$. The latter curve was multiplied by 15 to fit the total distribution at $\mathrm{P}_{\perp}=0.7$ a.u. 
Both calculations in Fig. 7 were performed for a hydrogen atom. However, one has to note that according to our calculations the shape of the momentum distribution is not very sensitive to the binding energy and weakly depends on the field intensity (within the interval of intensities considered). A similar conclusion is also suggested by Eq. (2) which predicts the width $(\mathrm{FWHM})$ of the spectrum to be proportional to $\left(\mathrm{I} / 2 \mathrm{I}_{\mathrm{p}}\right)^{1 / 4}$. Measurements performed with different intensities showed that up to $1 \mathrm{PW} / \mathrm{cm}^{2}$ the experimental data exhibit a similar intensity dependence, though the absolute width given by Eq. (2) is much broader then the experimental results.

According to the SFA calculation ${ }^{43}$ the cusp basically originates from the singularity in the electron continuum wave function at zero energy in a Coulomb system, which is also present in the Coulomb-Volkov state. However, this singularity is a necessary but not sufficient condition for the cusp structure to appear. Indeed, after absorption of a certain number of laser quanta the electron would have a definite energy which only by coincidence could be (very close to) zero. Therefore, it is only after the averaging over the spatio-temporal profile of the laser pulse, which leads to the ponderomotive energy varying from zero to its maximal value (reached in the focal center in the middle of the pulse), that the electron spectrum becomes continuous and states with zero energy are effectively populated. Thus, within this model the non-monochromaticity of the energy spectrum of emitted electrons due to the spatio-temporal structure of the pulse is essential for the cusp formation.

Despite good overall agreement, substantial difference between theory and experiment as well as between two different calculations can be observed in the wings of the momentum distribution (Fig. 7). In order to explore the origin of this discrepancy, we exploit the fact that fully differential ionization yields have been measured. Fig. 8 compares the total transverse momentum distribution (solid line) with that for the electrons with longitudinal momenta larger than $2 \sqrt{U p}$, i.e. beyond the maximum classical drift momentum that an electron can acquire from the laser field. These are electrons which gained energy by being rescattered off the parent ion. In striking contrast to the low energy region, for rescattered electrons we observe a broad, relatively smooth transverse momentum distribution. Since the latter part (open circles, multiplied by 15) closely follows the tail of the total distribution for $\left|P_{x}\right| \geq 0.7$ a.u., we conclude that the change of the slope in this region, as well as the discrepancies with the calculations observed in Fig. 7, are due to electron rescattering. Indeed, the latter is not included at all in the SFA model ${ }^{43}$. Furthermore, though the CTMC-T calculations $^{32}$ include rescattering and thus give better agreement with the experiment for high $\mathrm{P}_{\mathrm{x}}$, they still underestimate the relative yield of the rescattered electrons compared to the results of a numerical solution of the TDSE. This explains the differences observed in Fig. 6 for different targets at large transverse momenta: it is known that the relative strength of the rescattering plateau depends on the shape of the scattering potential, and, as a matter of fact, the plateau is most pronounced in $\mathrm{Ne}^{15,20}$. The difference observed in the absolute number of counts for the two curves in Fig. 8 can be readily understood by taking into account the fact that electrons with longitudinal momenta larger than $2 \sqrt{U p}$ are not the only ones that had experienced a rescattering event. We expect that a more detailed analysis of the fully differential data will allow us to distinguish 'direct' and 'rescattered' electrons in the low-energy region. It can also provide information on the subtleties of the rescattering process and would be of particular importance for molecular structure imaging using diffraction of the rescattered electrons as was recently suggested ${ }^{44}$.

\section{CONCLUSIONS AND OUTLOOK}

We have performed kinematically complete experiments on single ionization of rare gas atoms by intense laser fields over a broad range of Keldysh parameters $(1.1>\gamma>0.29)$, covering the transition from the multiphoton to the tunnelling ionization. We have observed a smooth transition between these two processes and have shown that loworder ATI peaks with some target-dependent substructure can be resolved deep in the tunnelling regime. The peaks do not exhibit ponderomotive shifts and are less pronounced for ionization by few-cycle laser pulses. These are typical features for resonant-enhanced ionization, well-known for the case of much lower intensities. The importance of resonant enhancement and its connection to recollision phenomena was recently demonstrated for the plateau in the photoelectron spectra ${ }^{22-26}$. Taking into account latest results showing that recollision effects also manifest themselves at low electron energies ${ }^{21,32}$, we conclude that the structures presented in our data originate from a similar type of resonant process. However, a detailed description of the intermediate states involved, their relation to the field-free atomic levels and influence on the electron energy and angular distributions remains the subject of forthcoming analyses. 
In the transverse momentum distributions we have observed a sharp cusp-like structure at zero momentum. This singularity was observed in a broad range of intensities $\left(0.15-2.0 \mathrm{PW} / \mathrm{cm}^{2}\right)$ for different targets. Here we would like to mention that we observed the same structure for single ionization of $\mathrm{H}_{2}$ and $\mathrm{N}_{2}$ molecules. Distinguishable target dependent effects were found only in the wings of the transverse distributions, where a considerable fraction of electrons experienced a recollision with the parent ion. The comparison with theory showed that the observed singularity at zero transverse momentum originates from the long-range Coulomb interaction between an emitted electron and the remaining ion.

Our data for the transverse direction exhibit a striking similarity with momentum distributions obtained in ionization by relativistic highly-charged ion impact ${ }^{45,46}$. This indicates that the observed structure has the same origin, i.e., it originates from the singularity in the electron continuum wave function at zero energy in the presence of a Coulomb field, a fact that is well-known in the physics of ion-atom collisions ${ }^{47-49}$. Ionization of $\mathrm{He}$ by $1 \mathrm{GeV} / \mathrm{u} \mathrm{U}^{92+}$ ion impact has been described using the equivalent photon approximation ${ }^{45,50,51}$, where the electromagnetic field of the ion is interpreted by a broad-band virtual photon field. To the best of our knowledge, the present study is the first experimental observation of the Coulomb cusp for ionization with real photons. However, in a purely 'photon' representation of the laser field the cusp would not appear, since the monochromaticity of the energy spectrum of the emitted electrons limits the available final state phase space and thus removes the singularity, as was discussed above. Our measurements were performed for Keldysh parameters ${ }^{4} \gamma \leq 1.1$, and, despite clear multiphoton features observed in the data (Fig. 2-5), the low-energy electron continuum was efficiently populated. Thus, the intriguing question remains how the cusp evolves for lower intensities towards the "true" multiphoton regime.

\section{ACKNOWLEDGEMENTS}

The authors are grateful to A.B. Voitkiv, B. Najjari, K. Dimitriou, D. Arbo and S. Yoshida for providing the data of Fig. 7 and for many fruitful discussions.

\section{REFERENCES}

1 J.H. Eberly and J. Javanainen, Eur. J. Phys. 9, 265 (1991).

2 L.F. DiMauro and P. Agostini, Adv. At. Mol. Phys. 35, 79 (1995).

3 M. Protopapas, C.H. Keitel, and P.L. Knight, Rep. Prog. Phys. 60, 389 (1997).

${ }^{4}$ L.V. Keldysh, Zh. Exp. Teor. Fiz. 47, 1945 (1964) [Sov. Phys. JETP 20, 1307 (1965)

5 P. Agostini et al, Phys. Rev. Lett. 42, 1127 (1979).

${ }^{6}$ R.R. Freeman et al, Phys. Rev. Lett. 59, 1092 (1987).

7 G.N. Gibson, R.R. Freeman and T.J. McIlrath, Phys. Rev. Lett. 69, 1904 (1992).

8 M.P. de Boer and H.G. Muller, Phys. Rev. Lett. 68, 2747 (1992).

9 E. Mevel et al, Phys. Rev. Lett. 70, 406 (1993).

${ }^{10}$ R. Wiehle, B. Witzel, H. Helm, and E. Cormier, Phys. Rev. A 67, 063405 (2003).

${ }^{11}$ P.B. Corkum, N.H. Burnett and F. Brunel, Phys. Rev. Lett. 62, 1259 (1989).

${ }_{12}$ B. Delone and V. P. Krainov, J. Opt. Soc. Am. B 81207 (1991).

${ }^{13}$ N.B. Delone and V.P. Krainov, Phys. Usp. 41, 469 (1998).

${ }^{14}$ G.G. Paulus et al, Phys. Rev. Lett. 72, 2851 (1994).

15 B. Sheehy et al, Phys. Rev. A 58, 3942 (1998).

${ }^{16}$ Th. Weber et al, Phys. Rev. Lett. 84443 (2000).

${ }^{17}$ R. Moshammer et al, Phys. Rev. Lett. 84447 (2000).

${ }^{18}$ R. Moshammer et al, Phys. Rev. Lett. 91, 113002 (2003).

${ }^{19}$ V.L.B. de Jesus et al., J. Electr. Spectr. Rel. Phen. 141127 (2004).

${ }^{20}$ A Rudenko et al., J. Phys. B 37 L407 (2004).

${ }^{21}$ J. Chen and C.H. Nam, Phys. Rev. A 66053415 (2002).

${ }^{22}$ P. Hansch, M.A. Walker, and L.D. Van Woerkom, Phys. Rev. A 55, R2535 (1997).

${ }^{23}$ M.P. Hertlein, P.H. Bucksbaum, and H.G. Muller, J. Phys. B 30, L197 (1997).

${ }^{24}$ H.G. Muller, Phys. Rev. A 60, 1341 (1999).

${ }^{25}$ J. Wassaf, V. Véniard, R. Taïeb, and A. Maquet, Phys. Rev. A 67, 053405 (2003). 
${ }^{26}$ H.G. Muller, Phys. Rev. Lett. 83, 3158 (1999).

${ }^{27}$ U. Mohideen et al, Phys. Rev. Lett. 71, 509 (1993).

${ }^{28}$ J. Ullrich et al, Rep. Prog. Phys. 66, 1463 (2003).

${ }^{29}$ V.L.B. de Jesus et al, J. Phys. B 37, L161 (2004).

${ }^{30}$ F. Grasbon et al, Phys. Rev. Lett. 91, 173003 (2003).

${ }^{31}$ A.. Alnaser et al, Phys. Rev. A 70023413 (2004).

${ }^{32}$ K. Dimitriou et al, Phys. Rev. A 70 061401(R) (2004).

${ }^{33}$ E. Cormier et al, J. Phys. B 34, L9 (2001).

${ }^{34}$ G.L. Yudin and M.Yu. Ivanov, Phys. Rev. A 63033404 (2001).

${ }^{35}$ F.H.M. Faisal, J. Phys. B 6 L89 (1973).

${ }^{36}$ H. Reiss, Phys. Rev. A 221786 (1980).

${ }^{37}$ J.Z. Kaminski, A. Jaron and F. Ehlotzky, Phys. Rev. A 53 1756(1996)

${ }^{38}$ D. Milosevic and F. Ehlotzky, Phys. Rev. A. 583124 (1998)

${ }^{39}$ A. Becker et al., Phys. Rev. A. 64023408 (2001).

${ }^{40}$ S. Basile, F.Trombetta and G. Ferrante, Phys. Rev. Lett. 612435 (1988).

${ }^{41}$ G. Duchateau, E. Cormier and R. Gayet, Eur. Phys. J. D 11191 (2000).

${ }^{42}$ M. Weckenbrock et al., Phys. Rev. Lett. 91123004 (2003).

${ }^{43}$ A. Rudenko et. al, J. Phys. B, in press (2005).

${ }^{44}$ M. Spanner, O. Smirnova, P.B. Corkum and M.Yu. Ivanov, J. Phys. B 37 L243 (2004).

${ }^{45}$ R. Moshammer et al, Phys. Rev. Lett. 79, 3621 (1997).

${ }^{46}$ A.B. Voitkiv and B. Najjari, J. Phys. B 374831 (2004).

${ }^{47}$ J. Macek, Phys. Rev. A 1235 (1970).

${ }^{48}$ J. Burgdörfer, Phys.Rev. Lett. 51374 (1983).

${ }^{49}$ J. Burgdörfer, M.Breinig, S.B. Elston and I.A. Sellin, Phys.Rev. A. 283277 (1983).

${ }^{50}$ C.F. Weizsäcker, Z. Phys. A $\mathbf{8 8} 612$ (1934).

${ }^{51}$ E.J. Williams, Phys. Rev. 45729 (1934). 